\title{
Hybrid Particle Cluster CPFD Simulation in the Acceleration and Stabilized Sections of a Downflow Circulating Fluidized Bed
}

Cesar Medina-Pedraza, Hugo de Lasa

Cesar Medina-Pedraza, Hugo de Lasa*

Chemical Reactor Engineering Centre, Department of Chemical and Biochemical Engineering, The University of Western Ontario, London, Ontario, N6A 3K7, Canada.

Email: hdelasa@uwo.ca

\begin{abstract}
This study reports a numerical hybrid cluster simulation of a downer reactor unit using the software CPFD Barracuda VR. This model includes an experimentally determined particle cluster size distribution, to describe the particle cluster morphological changes as well as the cluster acceleration. Gas-solid flows are considered for an FCC (Fluid Catalytic Cracking) catalyst with an apparent particle density of $1722 \mathrm{~kg} / \mathrm{m}^{3}$, flowing in a $5.08 \mathrm{~cm}$ I.D column with air at $300 \mathrm{~K}$ and with a pressure close to atmospheric. Conditions for the simulations consist of $1.0-1.6 \mathrm{~m} / \mathrm{s}$ superficial gas velocity and $30-50 \mathrm{~kg} / \mathrm{m}^{2} \mathrm{~s}$ solid mass flux ranges. Calculations developed involve the Hölzer-Sommerfeld and the Ganser Drag Correlations. The proposed numerical model is validated by comparing experimental and simulated radial cluster particle velocities and particle volume fractions. Simulations developed show that the Hölzer-Sommerfeld drag function in conjunction with a proper selection of cluster geometry provide good cluster velocities and particle volume fractions values both in the cluster acceleration and cluster stabilized flow unit sections.
\end{abstract}

\section{Introduction}

Downflow, or downer reactors are considered a viable and advantageous option to perform various catalytic reactions. It is anticipated that this operation mode can offer catalyst utilization enhancements when compared to the frequently utilized riser reactor. ${ }^{1}$ However, the downer reactor cannot be designed reliably using conventional scale-up methods, due to the complexity of the multiphase flow. Thus, and in order to implement this unit at an industrial scale, a physicochemical based method is required, to increase the reliability of the proposed design. ${ }^{2}$ 
Regarding the hydrodynamics in downer units, previous studies have shown a significant deviation of the particle velocity from the single particle velocity model. ${ }^{3}$ This consistent deviation, occurring with higher particle velocities than anticipated, led to envisioning solids flowing in downers as particle clusters. These particle clusters are formed as a result of several contributing factors such as (a) turbulence; (b) cohesive interparticle forces; (c) viscous stresses; (d) energy dissipation; and (e) collision between particles. ${ }^{4}$

In addition to the clustering phenomena, one can identify three main unit sections: (a) the first acceleration section; (b) the second acceleration section; and (c) the fully developed flow section. Clusters present in the developed flow region, are mainly formed by axially aligned particles, agglomerated in the form of strands. 5,6

Computational fluid dynamics (CFD) is a powerful tool to obtain approximate solutions for complex systems of equations. CFD is valuable to clarify physical behavior in gas-solid flows. The last three decades have seen an increased acceptance of CFD modeling for the unit design, the scale-up and the optimization of multiphase reactors. ${ }^{7}$ However, there are still theoretical and practical issues in the implementation of CFD, given that multiphase flows are complex, and that the available computational capacity is insufficient to simulate scaled-up units, due to the high particle population. ${ }^{8}$

One can identify the main approaches used in CFD, such as the Eulerian Continuum Framework and the Lagrangian Trajectory Method. The Eulerian-Eulerian Model, also called the Two-Fluid Method, considers the gas and solid phases as interpenetrating continua, and averages their behavior by using a statistical procedure. ${ }^{9}$ This method has been used to model the downer reactor previously. ${ }^{10,11}$ However, because particles cannot behave as a fluid, this modeling approach cannot lead to reliable results. Thus, for an in-depth understanding of the flow phenomena, other physical models are needed to account for gas-particle, particle-particle and particle-wall interactions. ${ }^{12}$

A valuable alternative is provided by the Lagrangian Method, which is based on a moving framework following the particle trajectory. There are, in this respect, several important approaches which require different levels of modeling and computational effort, such as the Direct Numerical Simulation (DNS) and the CFD-Discrete Element Method (CFDDEM). ${ }^{13-15}$ However, these more rigorous methods are not practical for a large number 
of particle computations, as is the case with FCC industrial scale reactor units with trillions of particles to be accounted for. ${ }^{8}$

The Multiphase Particle-in-Cell (MP-PIC) Method involves a discrete particle method where the fluid phase is treated as a continuum and the solids are modeled using Lagrangian computational groups of particles, also called "clouds" or "computational parcels". ${ }^{16}$ This approach reduces the computational effort while still accounting for particle interactions. The software package CPFD Barracuda VR implements the MP-PIC method providing three-dimensional simulations.

Abbasi et al. ${ }^{17}$ described downer hydrodynamics in three dimensions by using the CPFD Barracuda VR software. It was observed that in downers, there is a "wavy" flow with solid densification, leading to high solid fraction regions followed by low solid fraction regions. This "wavy" flow is more apparent at the end of the downer unit, with circumferential gas flow being promoted. The proposed model was considered as adequate, given that the fluid dynamic flow predictions passed several numerical tests.

Lanza et al. ${ }^{18,19}$ implemented a hybrid numerical model in CPFD Barracuda VR, by using the data from experimental cluster size distributions in two downers of different crosssectional areas. This model designated as the "Hybrid Experimental Numerical Cluster" model permitted an accurate prediction of solid-phase properties in the developed flow sections of both downers. The results of these authors also indicated a smaller near-wall influence for a downer with a diameter of $0.051 \mathrm{~m}$ compared to one with a diameter of $0.0254 \mathrm{~m}$.

One should note however, that despite earlier downer studies reported in the technical literature, particle cluster characteristics and dynamics in the first and second downer acceleration regions have not been modeled. Thus, the CPFD simulations of the present study are original, given that they clarify issues of critical importance for the design of the feeding sections of downer units, as well as for their operation. 


\section{Experimental Data}

\subsection{Catalyst}

The solid particles selected in the present study, are Fluid Catalytic Cracking (FCC) catalyst particles with an apparent particle density of $1722 \mathrm{~kg} / \mathrm{m}^{3}$. The particles have a mean diameter of $84.4 \mu \mathrm{m}$ and standard deviation of $33.6 \mu \mathrm{m}$.

\subsection{Downer Geometry}

The chosen downer geometry is a replica of an available experimental system, consisting of a cyclone, an air distributor, and a column of $0.051 \mathrm{~m} \mathrm{ID}$ and $2 \mathrm{~m}$ in height. Details of the selected downer unit are provided in Figure 1.

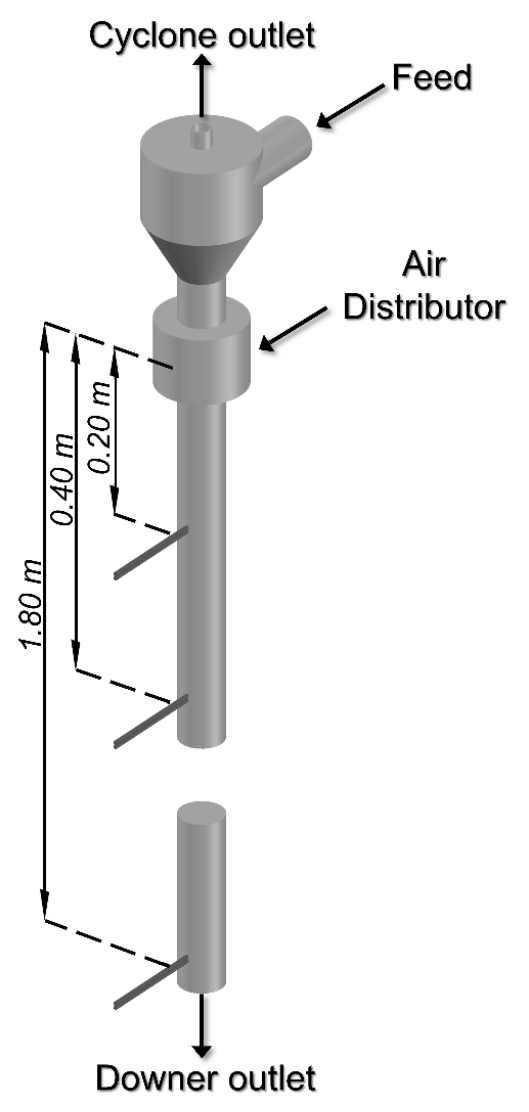

Figure 1. Dimensions of the Experimental Downer Unit Selected for the CPFD Simulations Figure 1 also describes the various axial levels at which the experimental measurements were taken with the CREC-Optiprobes. Additional information about the experimental system is provided in Medina and de Lasa. ${ }^{20}$ 


\section{Modeling}

The numerical model was validated with the experimental data previously obtained. This was done by using CPFD Barracuda VR simulations. This model uses the control volume formulation with staggered scalar and momentum nodes, to approximate the discretized conservation equations for the fluid and solid phases.

\subsection{Fluid Phase Equations-Continuous Phase}

The fluid mass and momentum equations for dense particle flow, using MP-PIC, are given by Equations 1 and 2:

$$
\begin{gathered}
\frac{\partial \varepsilon_{f} \rho_{f}}{\partial t}+\nabla\left(\varepsilon_{f} \rho_{f} u_{f}\right)=0 \\
\frac{\partial\left(\varepsilon_{f} \rho_{f} u_{f}\right)}{\partial t}+\nabla \cdot\left(\varepsilon_{f} \rho_{f} u_{f} u_{f}\right)=-\nabla p-F+\varepsilon_{f} \rho_{f} g+\nabla \cdot\left(\varepsilon_{f} \tau_{f}\right)
\end{gathered}
$$

where $u_{f}$ is the fluid velocity; $\varepsilon_{f}$ is the fluid volume fraction; $\rho_{f}$ is the fluid density; $\tau_{f}$ is the fluid stress tensor; and $F$ is the interphase momentum transfer.

\subsection{Solid Phase Equations-Discrete Phase}

For the particle field, a particle distribution function, $f$, is considered. $f$ is function of particle spatial location, $\mathrm{x}$, particle mass $m_{p}$, particle velocity $u_{p}$, and time t: $f\left(x, u_{p}, m_{p}, t\right)$. The transport equation for $f$ is given by Equations 3 and 4 :

$$
\begin{gathered}
\frac{\partial f}{\partial t}+\frac{\partial\left(f u_{p}\right)}{\partial x}+\frac{\partial\left(f \dot{u}_{p}\right)}{\partial u_{p}}=\left(\frac{\partial f}{\partial t}\right)_{c o l l}^{D} \\
\dot{u}_{p}=\frac{d u_{p}}{d t}=D_{s}\left(u_{f}-u_{p}\right)-\frac{1}{\rho_{s}} \frac{\partial p}{\partial x}-\frac{1}{\varepsilon_{s} \rho_{s}} \frac{\partial \tau}{\partial x}+g
\end{gathered}
$$

Where $\dot{u}_{p}$ is the particle acceleration; $\varepsilon_{s}$ is the particle volume fraction; $\rho_{s}$ is the solid material density; $p$ is the fluid pressure; $g$ is the gravity acceleration; $\tau$ is the particle contact stress. $D_{s}$ is the drag function which is defined by Equation 5 as follows:

$$
D_{s}=\frac{3}{8} C_{D} \frac{\rho_{f}\left|u_{f}-u_{p}\right|}{\rho_{s} r_{p}}
$$


The right-hand side of Equation 3 relates to the collision damping term defined by Equation 6 as follows:

$$
\left(\frac{\partial f}{\partial t}\right)_{\text {coll }}^{D}=\frac{f_{D}-f}{\tau_{D}}
$$

where $\tau_{D}$ is the collision damping time; and $f_{D}$ is the probability distribution function obtained by collapsing the velocity dependence of $f$ to a delta function centered at the local mass-averaged particle velocity, $\overline{u_{p}}$ given by Equation 7 :

$$
f_{D}=\left[\int f d u_{p}\right] \delta\left(u_{p}-\overline{u_{p}}\right)
$$

Additional information about the collision damping term and the particle and fluid transport equations can be found in O'Rourke and Snider ${ }^{21}$ and Snider. ${ }^{16}$

Furthermore, and considering the $f$ particle distribution function, the gas phase volumetric fraction is calculated as per Equation 8:

$$
\varepsilon_{f}=1-\frac{1}{V_{\text {cell }}} \iint f \frac{m_{p}}{\rho_{p}} d m_{p} d u_{p}
$$

As well, the interphase momentum transfer function introduced in Equation 2 is defined with $f$ by Equation 9 as follows:

$$
F=\iint f m_{p}\left[D_{s}\left(u_{f}-u_{p}\right)-\frac{1}{\rho_{s}} \nabla p\right] d m_{p} d u_{p}
$$

\subsection{Hybrid Experimental Numerical Cluster Model}

Particles evolve in a downer unit by going through a process of acceleration and stabilization. At the entrance of the downer, it is hypothesized that particles aggregate, forming clusters of a quasi-spherical shape, while packed with a particle voidage close to the one of minimum fluidization, designated as $\varepsilon_{m f}$. Equations 10-12 describe such a cluster as follows:

$$
V_{c, \text { sphere }}=\varepsilon_{m f} \frac{1}{6} \pi(L)^{3}
$$




$$
\begin{gathered}
A_{c, \text { sphere }}=\frac{\pi}{4}(L)^{2} \\
L=N * d_{p}
\end{gathered}
$$

Where $L$ is the axial length of a cluster; and $N$ is the number of particles with an average diameter, $d_{p}$, in the axial direction.

On the other hand, when clusters stabilize inside the downer, they display a strand or a "train of particles" shape. Thus, the cluster volume can be calculated with Equation 13 as follows:

$$
V_{c, \text { strand }}=\frac{1}{6} \pi d_{p}^{3} N
$$

As well, the strand shaped cluster cross-sectional area normal to the flow can be defined with Equation 14 as follows:

$$
A_{c, \text { strand }}=\frac{\pi}{4} d_{p}^{2}
$$

One can notice that both particle configurations for the acceleration and stabilized region, may display the same axial length as shown in Figure 2, having however, a significantly different cluster volume.

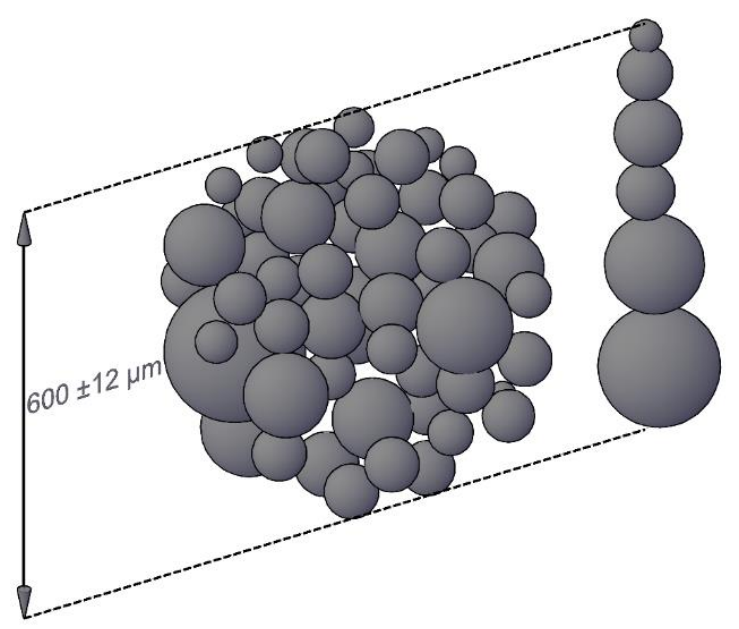

Figure 2. Particles Forming a Particle Cluster in Two Different Configurations: Spherical and Strand Shape

As discussed in a recent article, ${ }^{20}$ experimental measurements at various axial positions, were obtained with the CREC-GS-Optiprobes. ${ }^{22}$ These measurements allow recording 
both individual cluster velocities and particle volume fractions. An example of the axial cluster size distribution obtained experimentally at both the entry and stabilized downer regions is shown in Figure 3.

a)

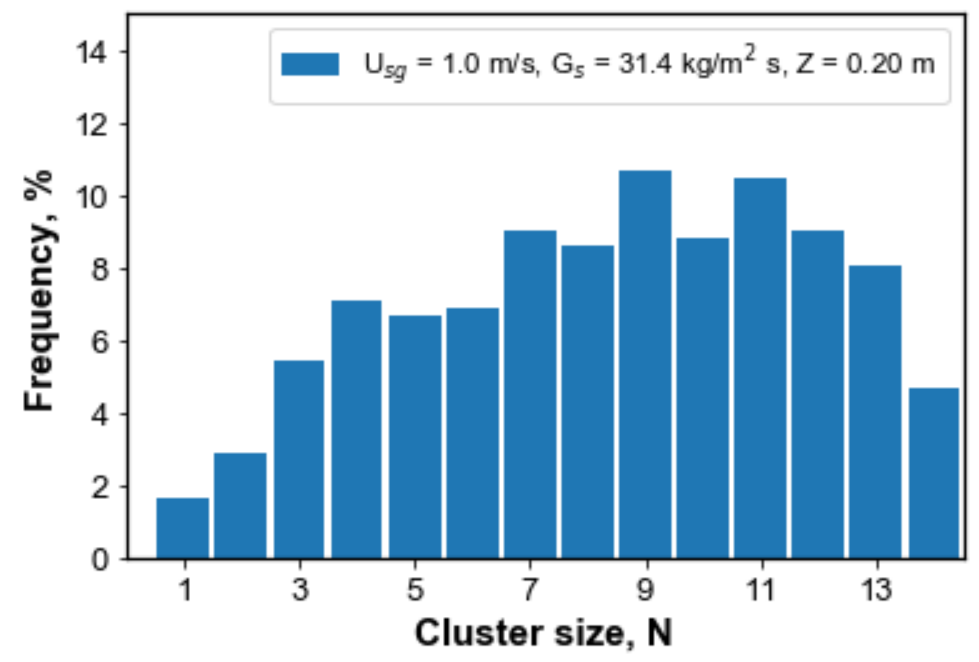

b)

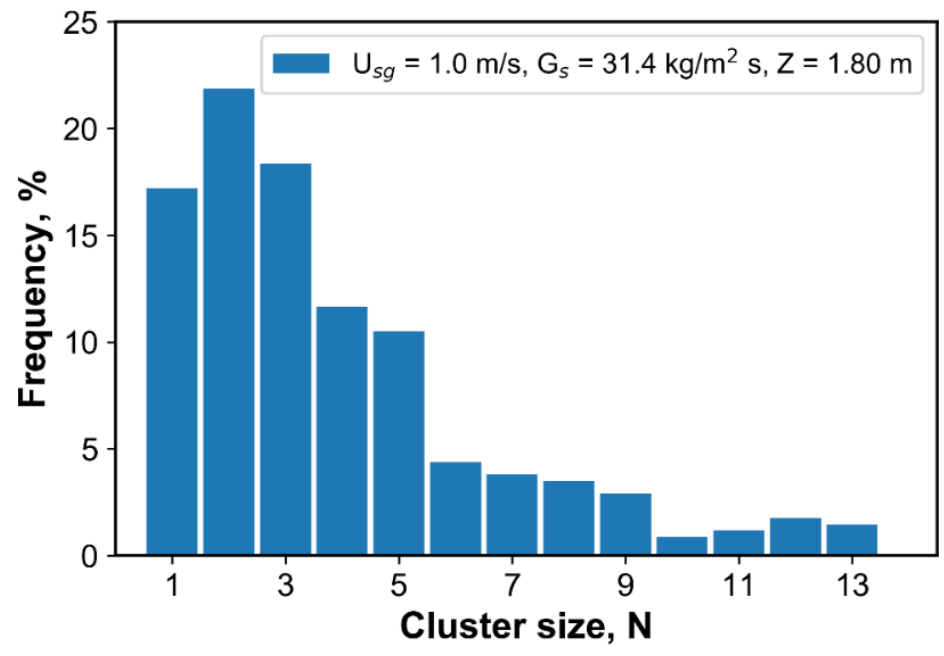

Figure 3. Cluster Size Distribution at $U_{s g}=1.0 \mathrm{~m} / \mathrm{s}, G_{s} \approx 31 \mathrm{~kg} / \mathrm{m} 2 \mathrm{~s}$ for (a) $Z=0.20 \mathrm{~m}$, (b) $Z=$ $1.80 \mathrm{~m}$

As claimed by Lanza et al. ${ }^{18}$, these data, such as axial particle cluster size distributions are valuable, given that they can be considered into hybrid CPFD Barracuda VR numerical simulations.

\subsection{Drag Correlation}

The particle cluster drag coefficient is a critical parameter in downer simulations. In this respect, Chhabra et al. ${ }^{23}$ used an extensive experimental dataset and compared different methods for assessing particle cluster drag coefficients. In these studies, the Ganser correlation for non-spherical particles yielded the lowest error percentages for the expected configurations of the clusters in this system.

Based on these findings, Lanza ${ }^{19}$ selected the Ganser Drag Model for the calculations reported in their work. The Ganser drag coefficient is calculated with equations 15 and 16 as follows:

$$
C_{D}=\frac{24}{R e_{p} K_{1} K_{2}}\left(1+0.1118\left(R e_{p} K_{1} K_{2}\right)^{0.6567}\right)+\frac{0.4305}{1+\frac{3305}{R e_{p} K_{1} K_{2}}}
$$




$$
K_{1}=\frac{3}{1+2 \phi^{-0.5}} ; K_{2}=10^{1.8148(-\log \phi)^{0.5743}}
$$

More recently, it was reported by Bagheri 24 that when using the Hölzer-Sommerfeld Model ${ }^{25}$, a lower error was obtained in the calculation of motion of non-spherical particles, as compared to when the Ganser Correlation was used. The Hölzer-Sommerfeld Correlation uses three shape parameters: (a) cross-wise sphericity, $\phi_{\perp}$; (b) standard sphericity, $\phi$; and (c) length-wise sphericity, $\phi_{\|}$. Consistent with this, the drag coefficient in the present work, is calculated according to Equation 17.

$$
C_{D}=\frac{8}{R e_{p} \sqrt{\phi_{\|}}}+\frac{16}{R e_{p} \sqrt{\phi}}+\frac{3}{\sqrt{R e_{p}} \phi^{0.75}}+0.42 * 10^{0.4(-\log \phi)^{0.2}}\left(\frac{1}{\phi_{\perp}}\right)
$$

The sphericity shape factors are described by the ratios presented in Equations 18-20.

$$
\begin{gathered}
\phi_{\|}=\frac{\text { cross }- \text { sectional area of the volume equivalent sphere }}{\frac{1}{2} \text { cluster surface area }- \text { cluster projected area, parallel to flow }} \\
\phi=\frac{\text { surface area of volume equivalent sphere }}{\text { cluster surface area }} \\
\phi_{\perp}=\frac{\text { cross }- \text { sectional area of the volume equivalent sphere }}{\text { cluster projected area, normal to flow }}
\end{gathered}
$$

As a result, and considering the potential shape change of particle clusters while evolving in downer units, the model given by Equations $17-20$ is considered in the simulations.

\section{Results and Discussion}

\subsection{Simulation Conditions}

Regarding numerical simulation and fluid dynamic modeling, the Hybrid ExperimentalNumerical model was applied to both the acceleration and the fully developed flow sections of the downer. This Hybrid Experimental-Numerical model uses the cluster axial size distribution determined experimentally as the particle size distribution in the simulations. As well, the considered model accounts for possible cluster shape, in order 
to establish the best particle cluster geometry to best fit the experimental data. Table 1 reports the various parameters considered using CPFD Barracuda VR.

Table 1. Input Parameters for Barracuda Simulation

\begin{tabular}{|c|c|c|}
\hline & Parameter & Value \\
\hline & Geometry & Figure 1 \\
\hline Heat transfer & Isothermal flow & $\mathrm{T}=300 \mathrm{~K}$ \\
\hline Gravity & Z component of gravity & $-9.81 \mathrm{~m} / \mathrm{s}^{2}$ \\
\hline \multirow[t]{3}{*}{ Fluid properties } & Density & $1.17285 \mathrm{~kg} / \mathrm{m}^{3}$ \\
\hline & Molecular weight & 28.9652 \\
\hline & Viscosity & $1.8495 \times 10-5 \mathrm{~Pa} \mathrm{~s}$ \\
\hline \multirow[t]{2}{*}{ Solid properties } & Density & $1722 \mathrm{~kg} / \mathrm{m}^{3}$ \\
\hline & Molecular weight & 101.961 \\
\hline \multirow{2}{*}{$\begin{array}{l}\text { Particle-to-particle } \\
\text { interaction }\end{array}$} & Close-pack volume fraction & 0.55 \\
\hline & $\begin{array}{l}\text { Maximum redirection from } \\
\text { collision }\end{array}$ & $60 \%$ \\
\hline \multirow[t]{2}{*}{ Particle-fluid interaction } & Drag model & $\begin{array}{l}\text { Hölzer-Sommerfeld } \\
\text { Ganser }\end{array}$ \\
\hline & Sphericity & Table 3 \\
\hline \multirow[t]{5}{*}{$\begin{array}{l}\text { Particle normal stress } \\
\text { model }\end{array}$} & Harris-Crighton model & \\
\hline & Ps constant & 1 \\
\hline & B constant & 3 \\
\hline & Eps constant & $1 e-8$ \\
\hline & Factor of average velocity & 0.8 \\
\hline \multirow[t]{3}{*}{$\begin{array}{l}\text { Particle-to-wall } \\
\text { interaction }\end{array}$} & $\begin{array}{l}\text { Normal-to-wall momentum } \\
\text { retention }\end{array}$ & 0.99 \\
\hline & $\begin{array}{l}\text { Tangent-to-wall momentum } \\
\text { retention }\end{array}$ & 0.99 \\
\hline & Diffuse bounce & 0 \\
\hline \multirow[t]{2}{*}{ Initial conditions } & Gas velocity & 0 \\
\hline & Gas pressure & $113100 \mathrm{~Pa}$ \\
\hline \multirow[t]{2}{*}{ Boundary conditions } & Outlet pressure & $113100 \mathrm{~Pa}$ \\
\hline & Particle feed & Table 3 \\
\hline
\end{tabular}




\begin{tabular}{lll} 
& Particle feed per average & 6000 \\
& volume, $n_{p}$ & \\
& Inlet air flow & Table 3 \\
\hline Solver settings & Maximum volume iteration & 10 \\
& Volume residual & $1 \mathrm{e}-7$ \\
& Maximum pressure iteration & 2000 \\
& Pressure residual & $1 \mathrm{e}-6$ \\
& Maximum velocity iteration & 50 \\
& Velocity residual & $1 \mathrm{e}-7$ \\
& Maximum energy iteration & 100 \\
& Energy residual & $1 \mathrm{e}-6$ \\
& Friction coefficient & 0.1 \\
\hline Time controls & Time step & $0.001 \mathrm{~s}$ \\
& End time & $20 \mathrm{~s}$
\end{tabular}

One should note, the values for the particle-wall interaction were chosen according to previous studies with similar systems. ${ }^{19,26-28}$ A sensitivity analysis was performed to evaluate the impact of the key parameter normal-to-wall momentum retention coefficient, the results can be found in the Supporting Information provided.

As mentioned above, the MP-PIC method used in CPFD Barracuda VR employs an Eulerian grid to solve the fluid phase equations and to interpolate with the solid phase. Furthermore, it is important to note that the $n_{p}$ value reported in Table 1 refers to the particle feed per average volume, set at the boundary condition. This value is estimated using Equation 21 as follows:

$$
n_{p}=\frac{N_{p}}{N_{\text {cells }} \varepsilon_{P}}
$$

where $N_{p}$ is the total number of computational particles in the system, $N_{\text {cells }}$ is the number of Eulerian cells which will be filled with particles at a volume fraction of $\varepsilon_{P}$.

In our view, a $n_{p}$ value larger than 6000 would mean longer and unjustified computational times. One can deduce this, given that Lanza et al ${ }^{18}$ demonstrated that for a downer unit operated under similar conditions, a value of 3000 was high enough to ensure result accuracy. 


\subsection{Grid Independence Test}

CPFD Barracuda VR uses a structured grid for the simulations. The accuracy of the numerical solutions of the differential equations depends on several factors. The two most important factors are the number of cells that constitute the mesh geometry, and the time step used to solve the various differential equations. As well, the Courant Flow Number (CFL) is another key parameter to consider in the numerical calculations. The CFL measures the flow of fluid and the number of particles moving through each cell, with a value below 1 being desirable. ${ }^{29}$

The computational time, which is the real time that is needed to complete a simulation, is proportional to the number of cells in the system and inversely proportional to the time step length. A strategy to obtain the most accurate results without consuming an excessive amount of computer time is to perform simulations calculating deviations at three different degrees of grid refinement: (a) coarse, (b) medium, and (c) fine. Thus, simulations are developed for each grid and errors are calculated. If this value is below $1 \%$, the grid is sufficiently accurate. For this case, the average fluid velocity is selected for the grid independence test.

$$
\begin{gathered}
\% \text { Error } 1=\frac{\text { Fluid Velocity }(\text { Medium })-\text { Fluid Velocity }(\text { Coarse })}{\text { Fluid Velocity }(\text { Medium })} 100 \\
\% \text { Error } 2=\frac{\text { Fluid Velocity }(\text { Fine })-\text { Fluid Velocity }(\text { Medium })}{\text { Fluid Velocity }(\text { Fine })} 100
\end{gathered}
$$

The dimensions of the grid used for the independence test as well as the corresponding errors at three axial positions are reported in Table 2.

Table 2. Grid Size and Grid Independence Test Results

\begin{tabular}{|c|c|c|c|c|c|c|}
\hline & Coarse & Medium & Fine & Error 1 & Error 2 \\
\cline { 2 - 7 } & $\begin{array}{c}\text { Grid size } \\
(\mathbf{m m})\end{array}$ & $10.24 \times 10.24 \times 20.76$ & $7.31 \times 7.31 \times 14.76$ & $5.68 \times 5.68 \times 10.93$ & & \\
\cline { 2 - 7 } & Cells & 4739 & 9355 & 17155 & & \\
\hline \multirow{2}{*}{$\begin{array}{c}\text { Fluid } \\
\text { velocity } \\
\text { (m/s) }\end{array}$} & $\mathbf{Z = 0 . 2 0}$ & -1.112 & -1.061 & -1.063 & -4.72 & 0.12 \\
\cline { 2 - 7 } & $\mathbf{Z}=\mathbf{0 . 4 0}$ & -1.123 & -1.062 & -1.059 & -5.76 & -0.27 \\
\hline \multirow{2}{*}{$\mathbf{m}=\mathbf{1 . 8 0}$} & -1.106 & -1.057 & -1.064 & -4.63 & 0.65 \\
\hline
\end{tabular}


According to the percentual errors reported in Table 2, the medium grid was considered accurate enough and, as a result, it was used in all ensuing reported simulations. The resulting feeding and aerial view of downer column sections of the grid are shown in Figure 4.
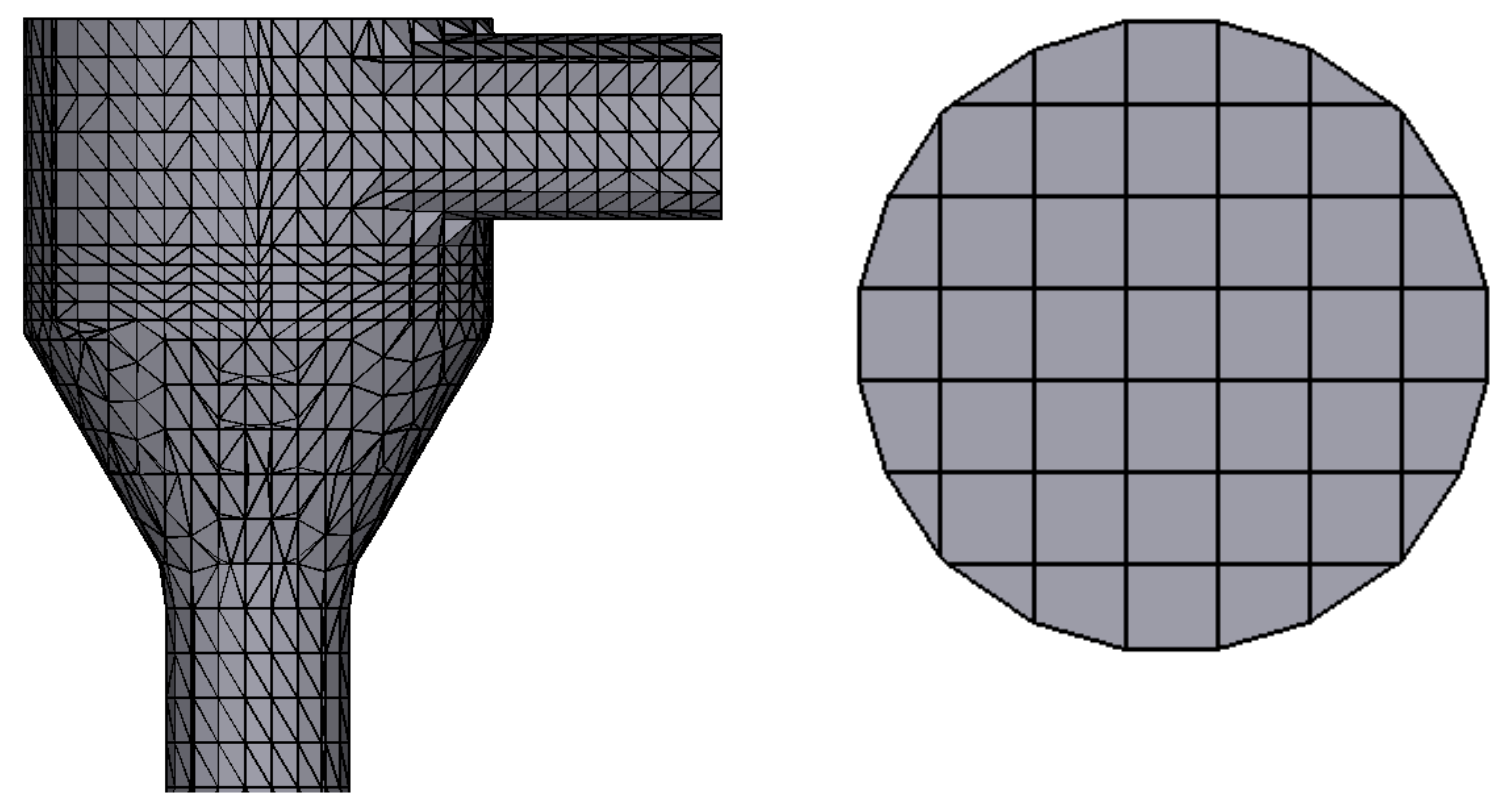

Figure 4. Medium Grid

\subsection{Boundary Conditions}

Four boundary conditions were specified in each simulation: (a) two mass flows, one for the cyclone inlet and one for the cyclone exit; (b) a pressure boundary condition for the downer outlet; and (c) the size and mass flow rate for each of the eight injection nozzles. These nozzles are distributed uniformly around the perimeter of the downer column, each nozzle has a diameter of $0.002 \mathrm{~m}$ and it is pointing towards the center with an inclination of 45 degrees. Additional information about the gas injector can be found in MedinaPedraza and de Lasa. ${ }^{20}$ The boundary conditions are depicted in Figure 5. 

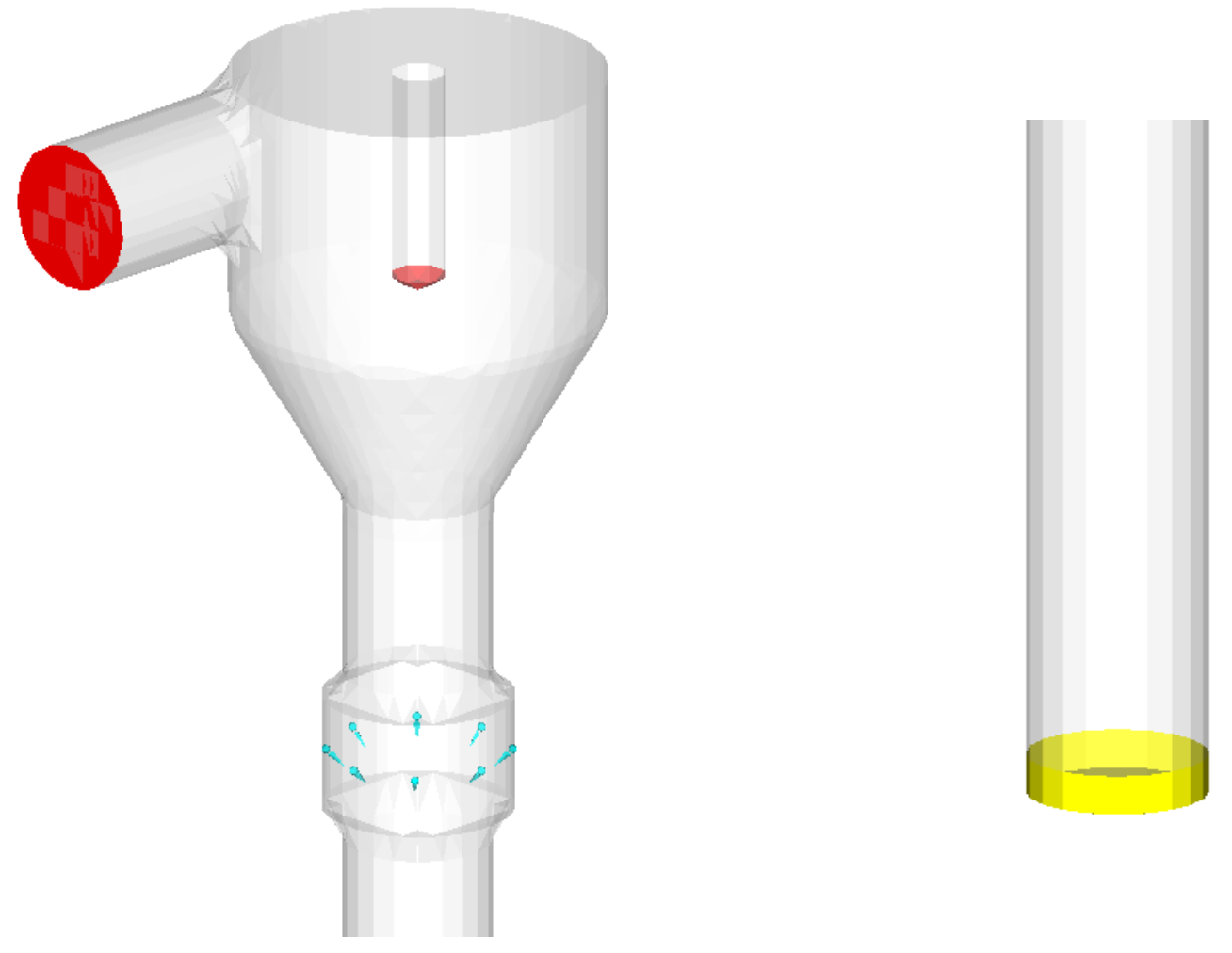

Figure 5. Boundary Conditions

The cyclone outlet and the injection mass flows were specified according to the conditions selected for the experiments, as reported in Medina Pedraza and de Lasa ${ }^{20}$. As well, the inlet solid mass flow was calculated by developing a mass balance which considers the determined outlet mass flow from the experiments, as proposed by Lanza et al. ${ }^{30}$

\subsection{Strategy for the Hybrid Model Evaluation}

Given that an important objective of this research is to evaluate the ability of the hybrid model to describe the downer operation in the acceleration (feeding) section, several methodological steps were considered: (a) the CREC-Optiprobes data from experiments were used to calculate cluster size distribution data; (b) the determined cluster size distributions were incorporated into the numerical downer model, to test its ability to predict particle cluster velocities and particle volumetric fractions.

\subsubsection{Cluster Configuration and Drag Correlation Evaluation}

In order to progress with the Hybrid Barracuda simulations, two different drag models originally proposed by Ganser ${ }^{31}$ and Hölzer-Sommerfeld ${ }^{25}$ were considered first, using the average cluster size distribution as reported in Table 3. 
As well, the drag correlation parameters were calculated for three different cluster shapes: (a) the cluster strand, constituted by a train of particles; (b) a prolate spheroid, with the minor semi-axis equal to half the length of the major semi-axis; c) a sphere, with the diameter equal to the cluster axial length recorded by the CREC-GS-Optiprobes.

The spheroid cluster mentioned above can be described with Equations 24-27, as follows:

$$
\begin{gathered}
V_{c, \text { spheroid }}=\varepsilon_{m f} \frac{4}{3} \pi a b^{2} \\
A_{c, \text { spheroid }}=\pi b^{2} \\
a=0.5 * N * d_{p} \\
b=0.5 * a
\end{gathered}
$$

The evaluation of these two drag models with the three shapes is reported in Figure 6, for three axial positions: $Z=0.20 \mathrm{~m}, Z=0.40 \mathrm{~m}$, and $Z=1.80 \mathrm{~m}$. Each simulation was run for 20 seconds and the results were obtained from the time-averaging of the last five seconds.

a) 

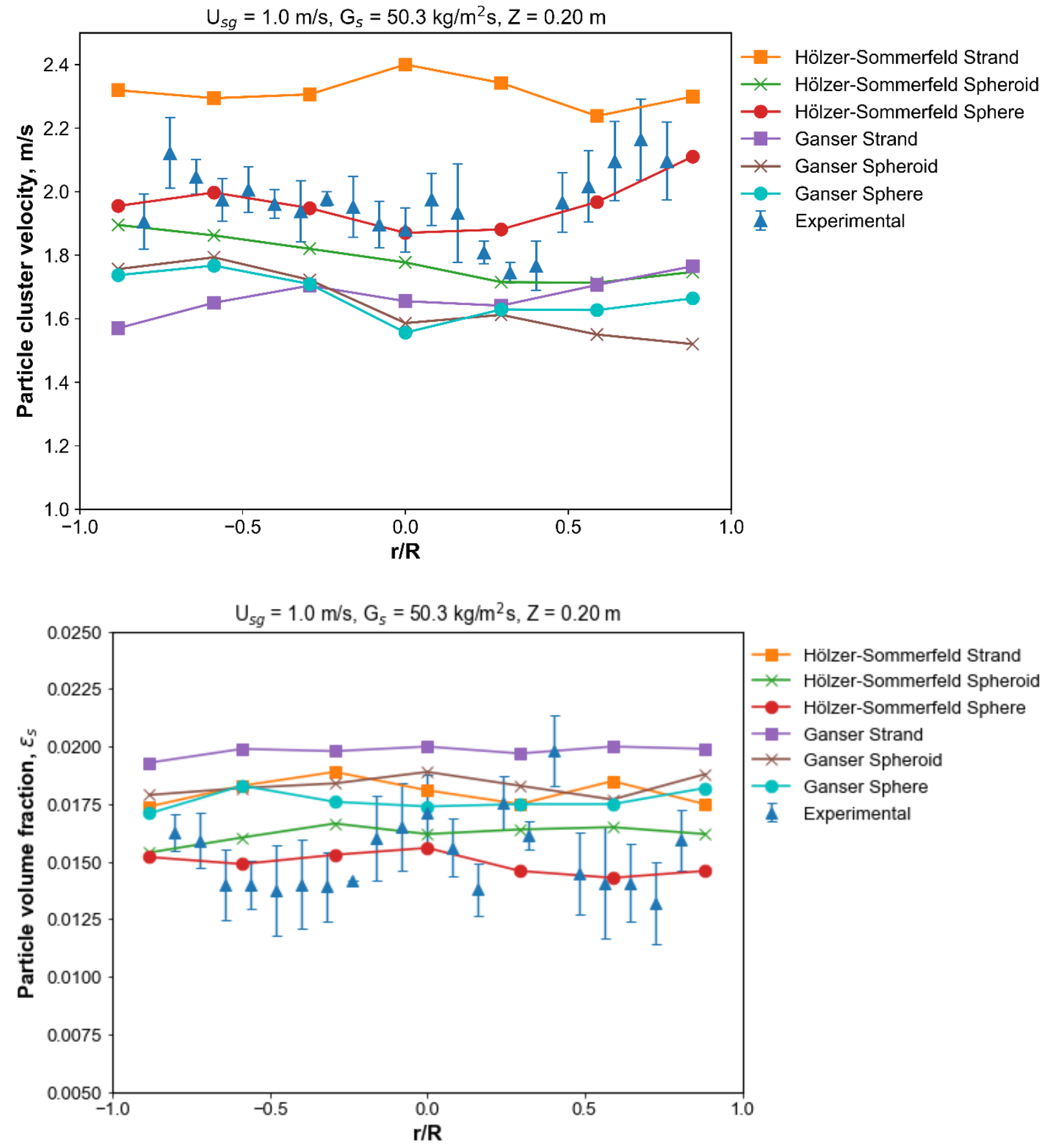
b)
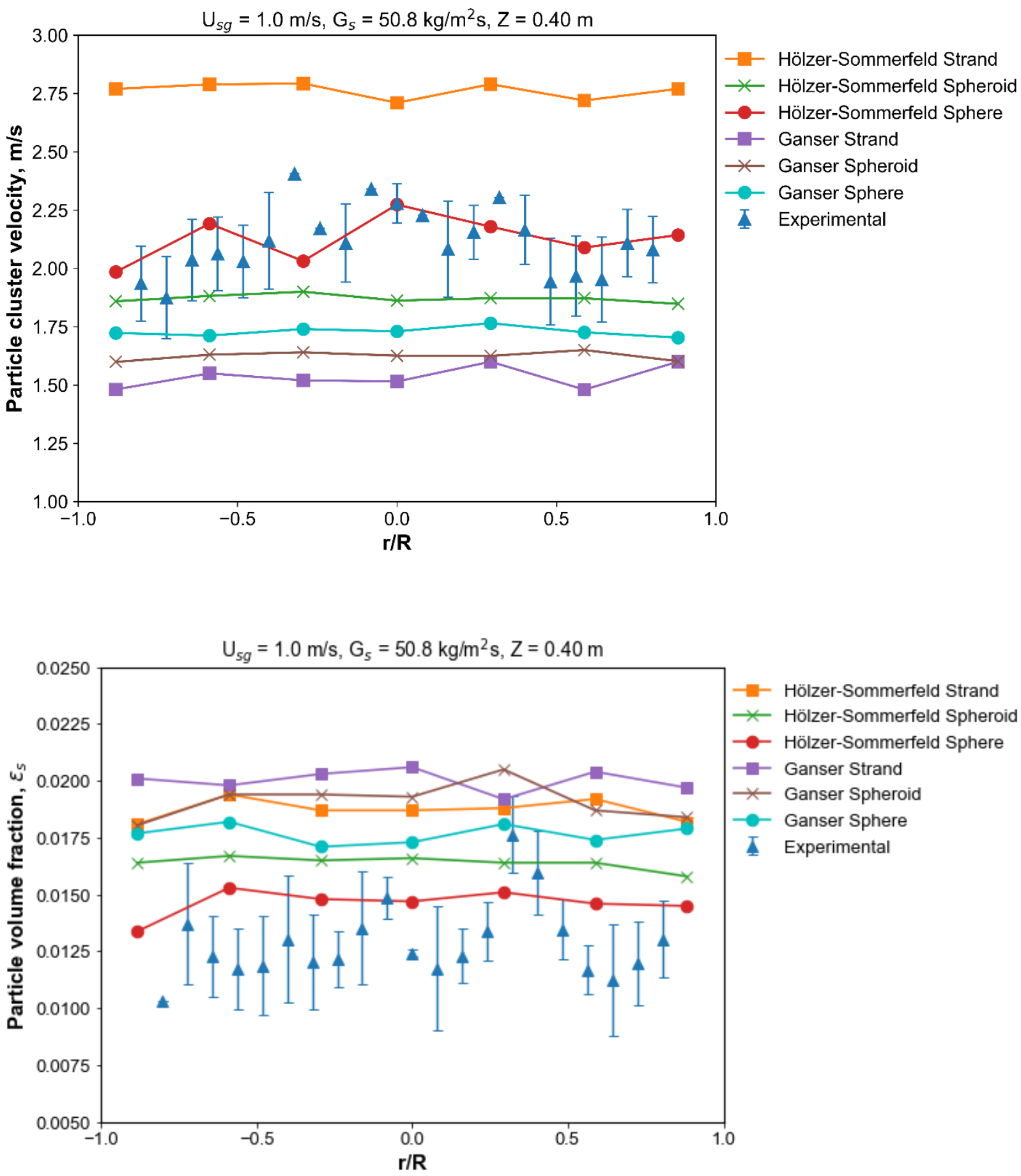

c) 

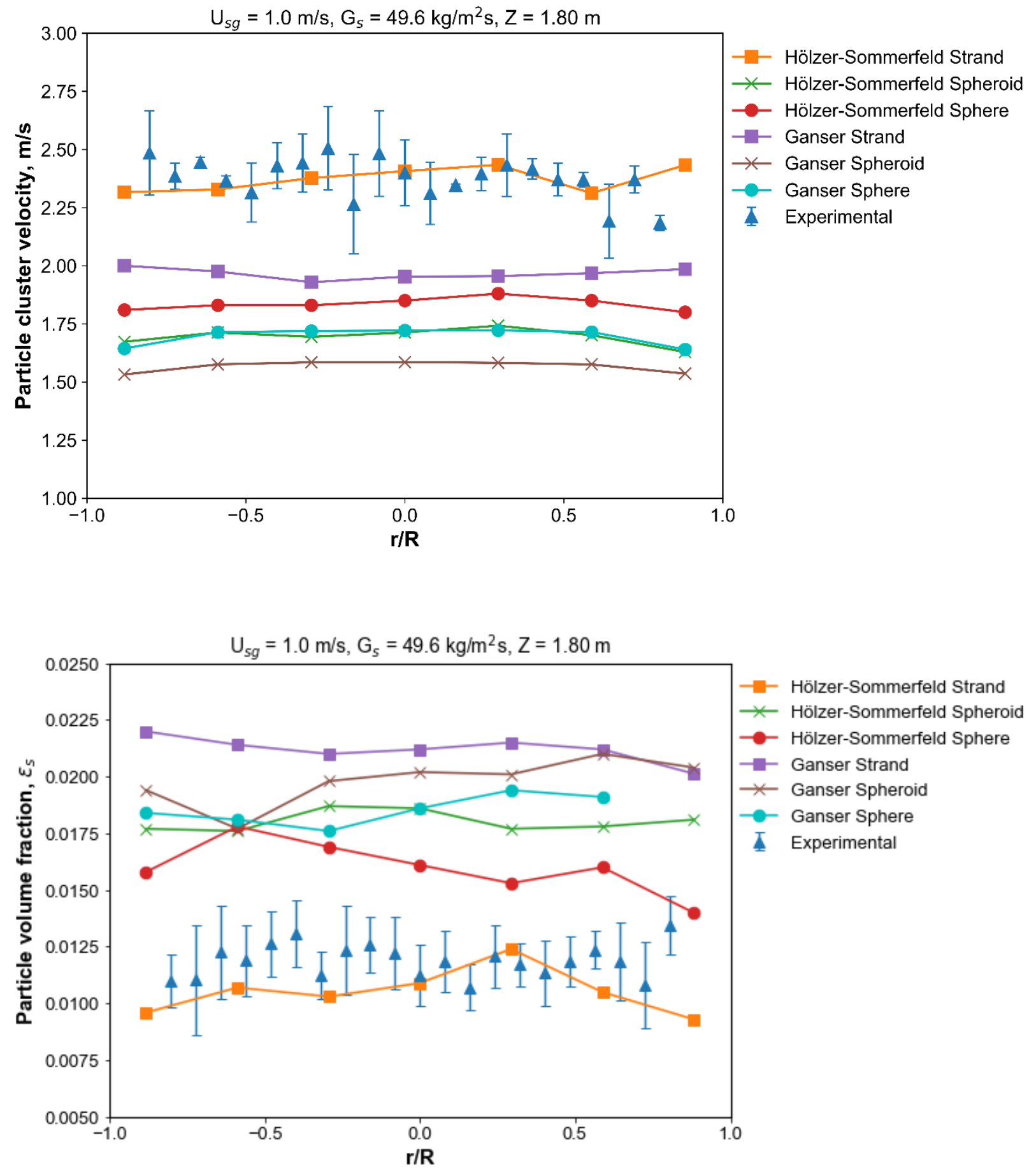

Figure 6. Radial Profiles of Particle Cluster Velocities and Particle volume fraction using HölzerSommerfeld and Ganser Drag Configurations at (a) $Z=0.20 \mathrm{~m}$, (b) $Z=0.40 \mathrm{~m}$, and (c) $Z=1.80$ $m$

On this basis, and from the particle cluster velocity radial profiles reported in Figure 6, it can be concluded that the Hölzer-Sommerfeld drag correlation provides the best 
approximation to the experimental results: (a) at the $0.20 \mathrm{~m}$ and $0.40 \mathrm{~m}$ axial positions when the spherical geometry is selected; and (b) at the $1.80 \mathrm{~m}$ axial position when the particle strand is chosen. Therefore, the Hölzer-Sommerfeld drag model is selected for subsequent simulations in the present study, using the spherical or strand geometries given that they are relevant to the axial positions under consideration.

Table 3 reports the operating conditions in terms of $U_{s g}$ and $G_{s}$ as well as the average axial cluster length and the sphericity parameters as required for the drag model. The spherical shape is assigned to clusters at $Z=0.20 \mathrm{~m}$ and $Z=0.40 \mathrm{~m}$ axial positions, while the strand shape is considered for clusters at $Z=1.80 \mathrm{~m}$.

Table 3. Operating Conditions and Average Cluster Characteristics for all Simulations

\begin{tabular}{|c|c|c|c|c|c|c|}
\hline Condition & $U_{s g}(m / s)$ & $\mathrm{G}_{\mathrm{s}}\left(\mathrm{kg} / \mathrm{m}^{2} \mathrm{~s}\right)$ & $N$ & $\phi_{\|}$ & $\phi$ & $\phi_{\perp}$ \\
\hline \multicolumn{7}{|c|}{$\mathrm{Z}=0.2 \mathrm{~m}$ from air distributor } \\
\hline 1 & 1.0 & 31.4 & 8.3 & 0.096 & 0.163 & 0.544 \\
\hline 2 & 1.0 & 50.3 & 8.7 & 0.091 & 0.156 & 0.544 \\
\hline 3 & 1.6 & 31.2 & 6.8 & 0.122 & 0.200 & 0.544 \\
\hline 4 & 1.6 & 53.7 & 7.1 & 0.113 & 0.187 & 0.544 \\
\hline \multicolumn{7}{|c|}{$\mathrm{Z}=0.4 \mathrm{~m}$ from air distributor } \\
\hline 5 & 1.0 & 30.2 & 5.3 & 0.139 & 0.230 & 0.672 \\
\hline 6 & 1.0 & 50.8 & 5.7 & 0.127 & 0.214 & 0.672 \\
\hline 7 & 1.6 & 30.5 & 4.6 & 0.165 & 0.265 & 0.673 \\
\hline 8 & 1.6 & 50.6 & 4.8 & 0.156 & 0.254 & 0.673 \\
\hline \multicolumn{7}{|c|}{$\mathrm{Z}=1.8 \mathrm{~m}$ from air distributor } \\
\hline 9 & 1.0 & 31.1 & 3.9 & 0.636 & 0.636 & 2.487 \\
\hline 10 & 1.0 & 49.6 & 4.1 & 0.627 & 0.627 & 2.558 \\
\hline 11 & 1.6 & 31.0 & 3.2 & 0.684 & 0.684 & 2.153 \\
\hline 12 & 1.6 & 52.0 & 3.3 & 0.672 & 0.672 & 2.230 \\
\hline
\end{tabular}

\subsection{Particle Cluster Velocity and Particle Volume Fraction Radial Profiles}

The next step in the study was the systematic evaluation of the Hybrid ExperimentalNumerical Cluster model in the acceleration and stabilized sections. To accomplish this, experimental results obtained previously in a cold-model downflow unit and reported in a previous article ${ }^{20}$ were used. In these experiments, the individual cluster size, the cluster velocity and the particle volume fraction were measured with the CREC-GS-Optiprobes. For all cases, the simulation was run for 20 seconds and the results were obtained from the time-averaging of the last five seconds. 
The validation of the Hybrid Barracuda Model is achieved as shown in Figures 7, 8 and 9 by comparing the simulation results with the experimental data. With this end in mind, radial particle cluster profiles for both cluster particle velocity and particle volume fraction are reported in the following manuscript sections.

a)
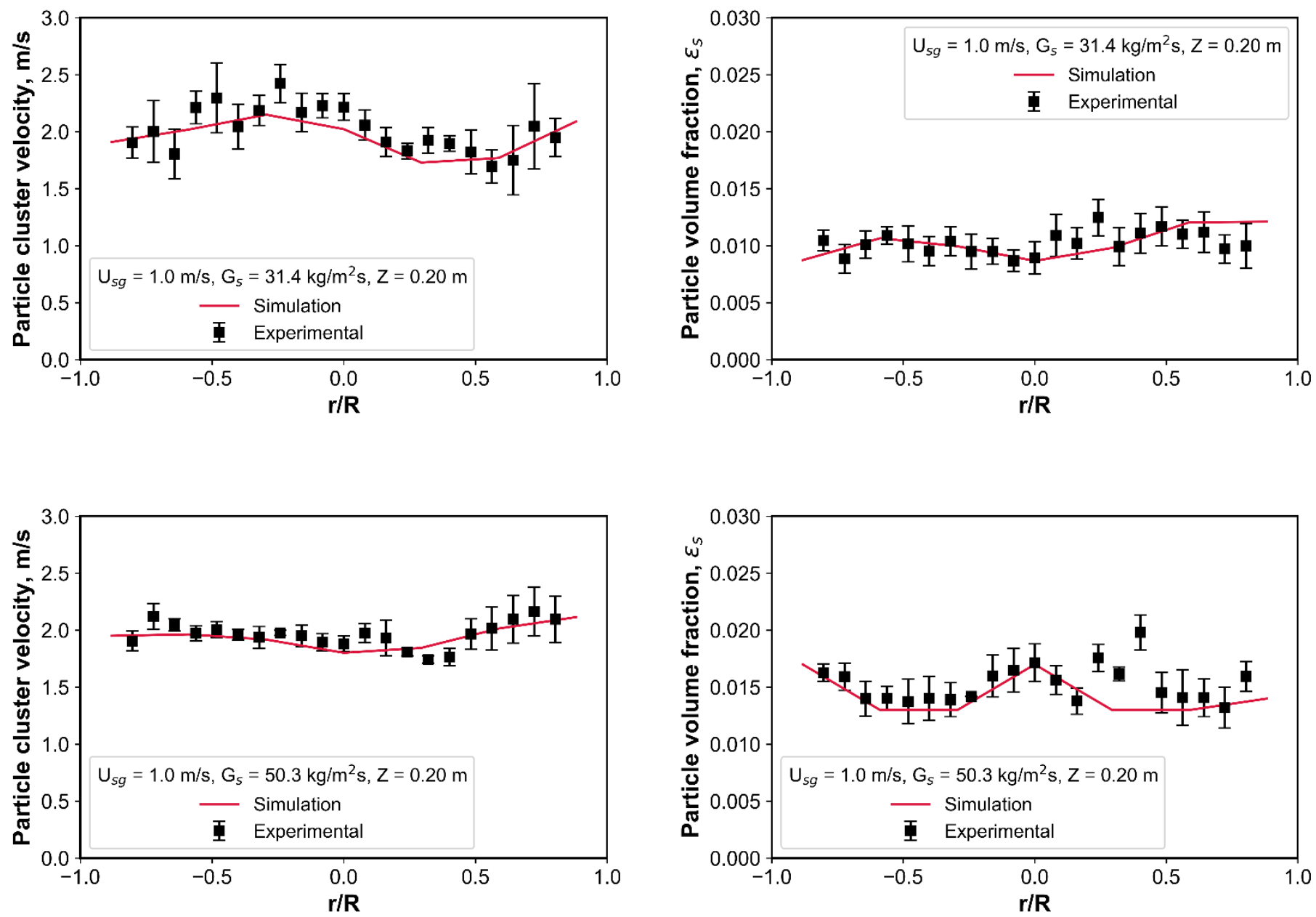

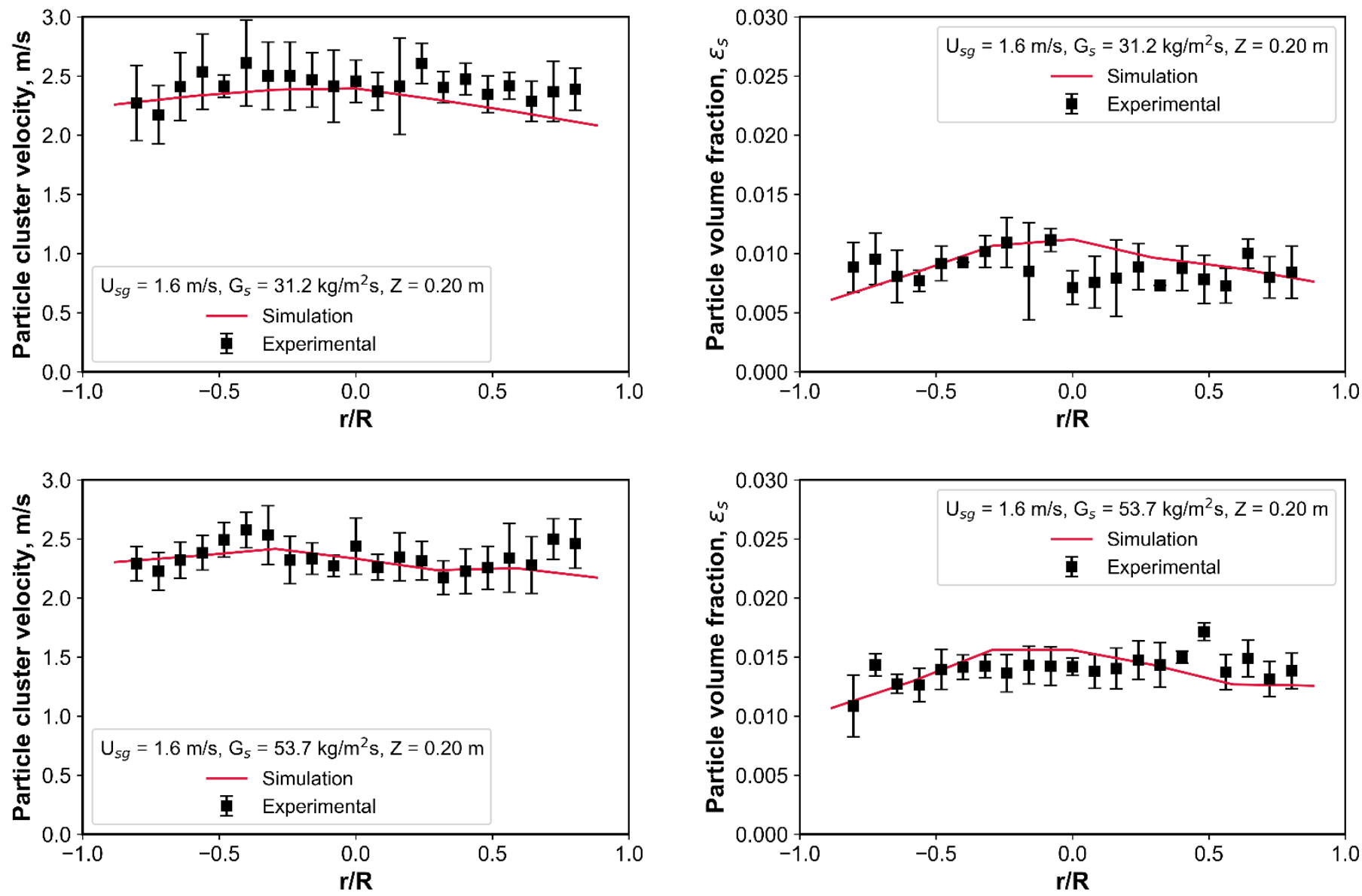

Figure 7. Radial Profiles of Cluster Velocities and Particle Volume Fractions at $Z=0.20 \mathrm{~m}$.

Particle Cluster Geometry: Spherical Shape.

Figure 7 reports cluster particle velocities and particle volumetric fractions for the proposed Hybrid Barracuda simulation using a Hölzer-Sommerfeld Drag Correlation and a spherical cluster geometry. One can see that the particle volume fractions and the cluster particle velocities both display close values in the Hybrid Barracuda simulations as well as in the experimental CREC-Optiprobe data. In addition, an interesting and consistent result which was found as well, was the lack of changes of these particle cluster velocities and particle volumetric fractions with radial position, remaining at close values, in all cases considered.

Figure 8 reports the further validation of the Hybrid Barracuda model with experimental data at the $\mathrm{Z}=0.40 \mathrm{~m}$ axial position and several radial downer positions. 

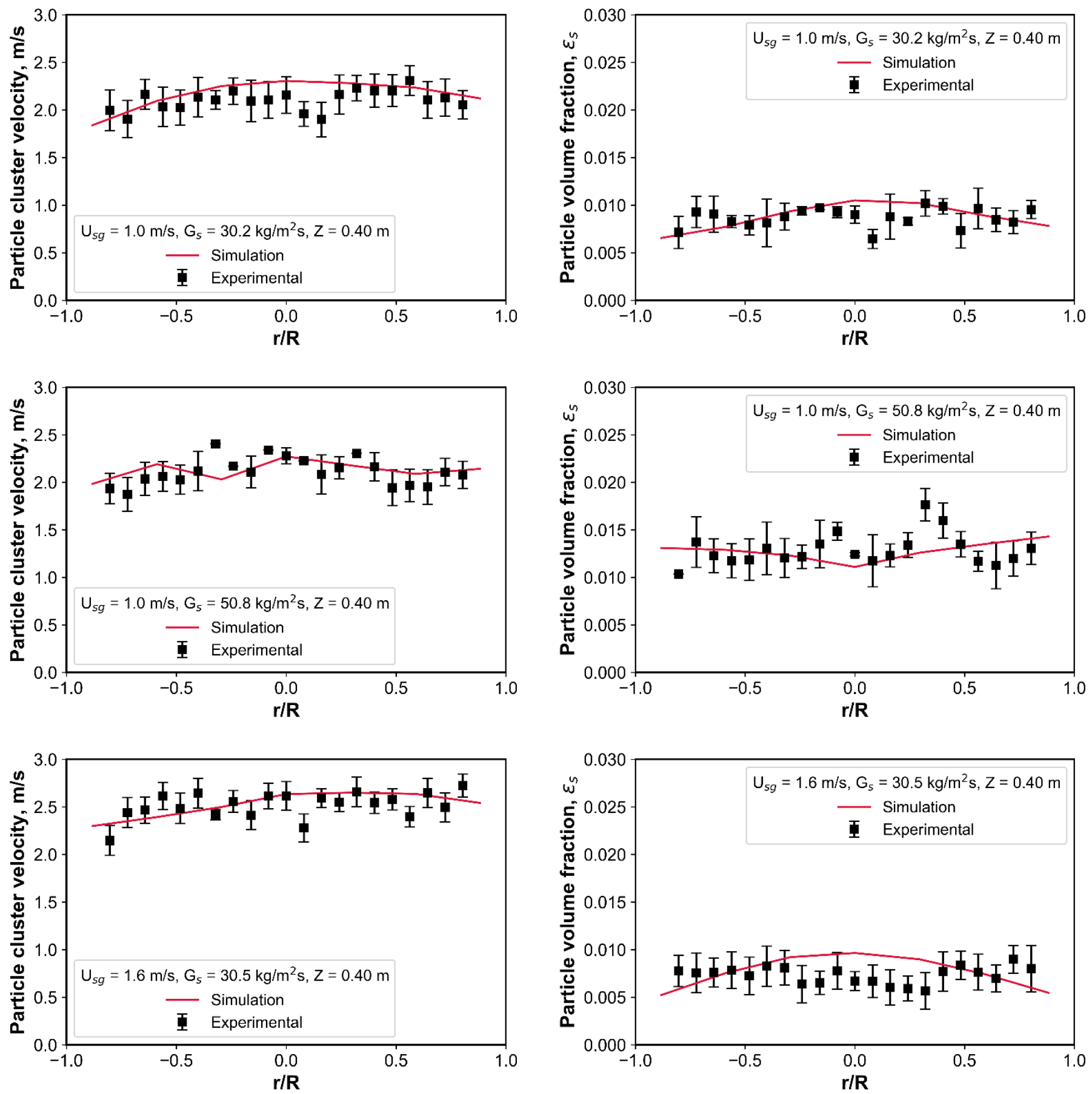

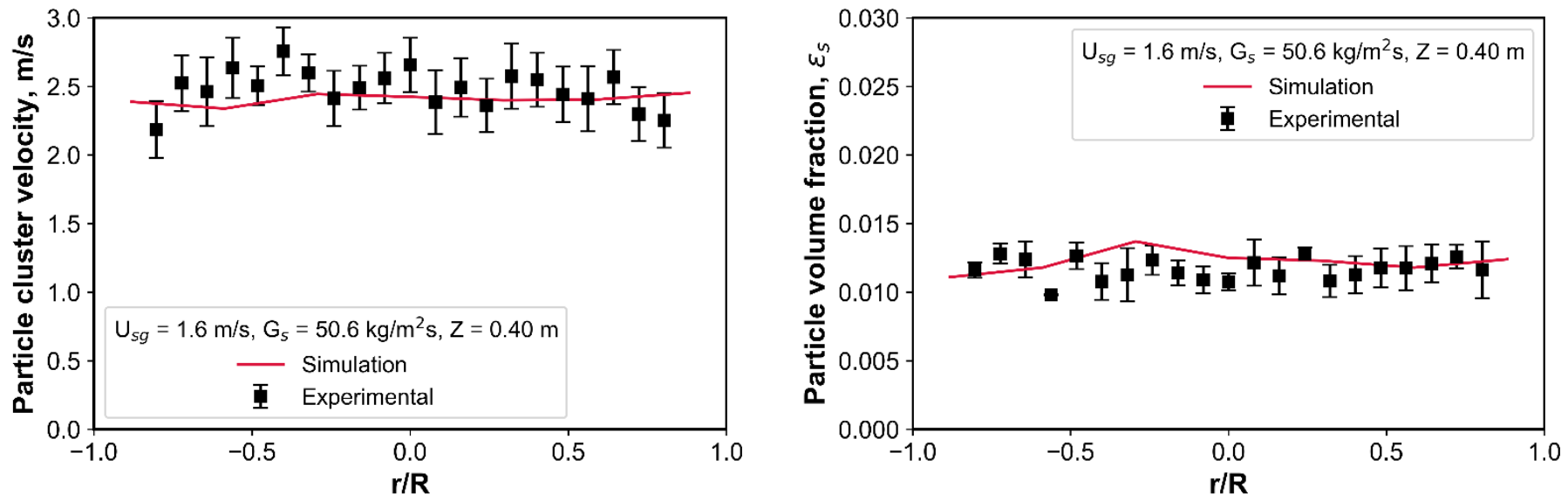

Figure 8. Radial Profiles of Cluster Velocities and Particle Volume Fractions at $Z=0.40 \mathrm{~m}$. Particle Cluster Geometry: Spherical Shape.

Figure 8 shows as well, the similarity between the Hybrid Barracuda Model simulations and the experimental values at various radial positions, if both the spherical geometry and the Hölzer-Sommerfeld Drag Correlation are selected for particle clusters.

Furthermore, Figure 9 revaluates the Hybrid Barracuda Model in the fully developed flow section, at the $Z=1.80 \mathrm{~m}$ axial position.
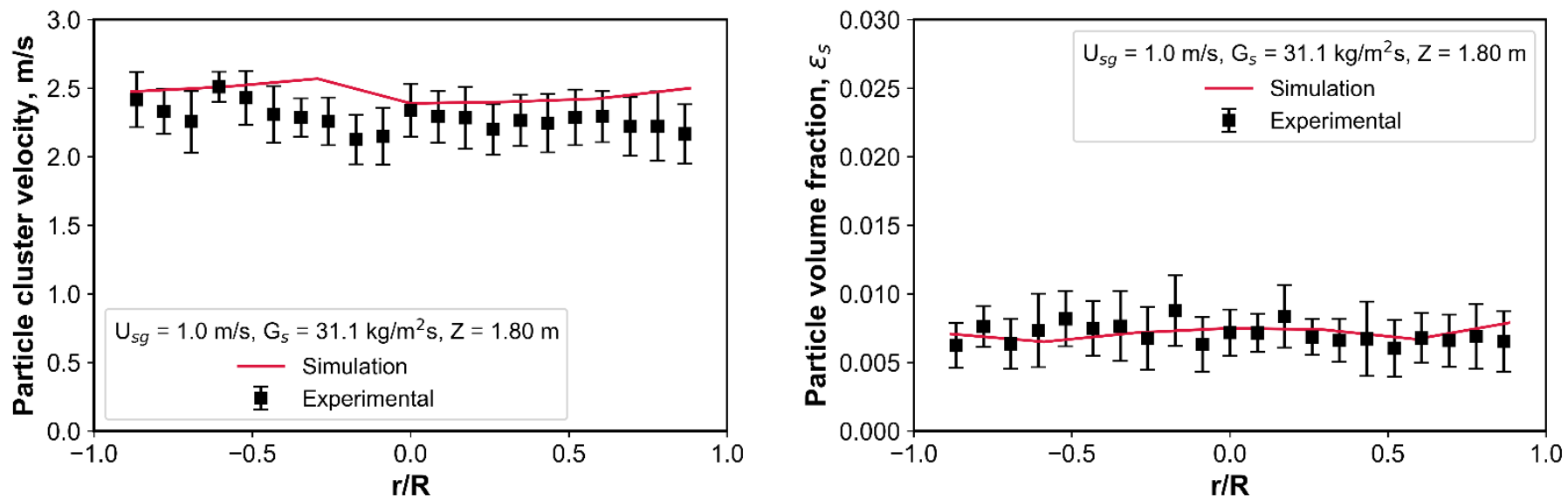

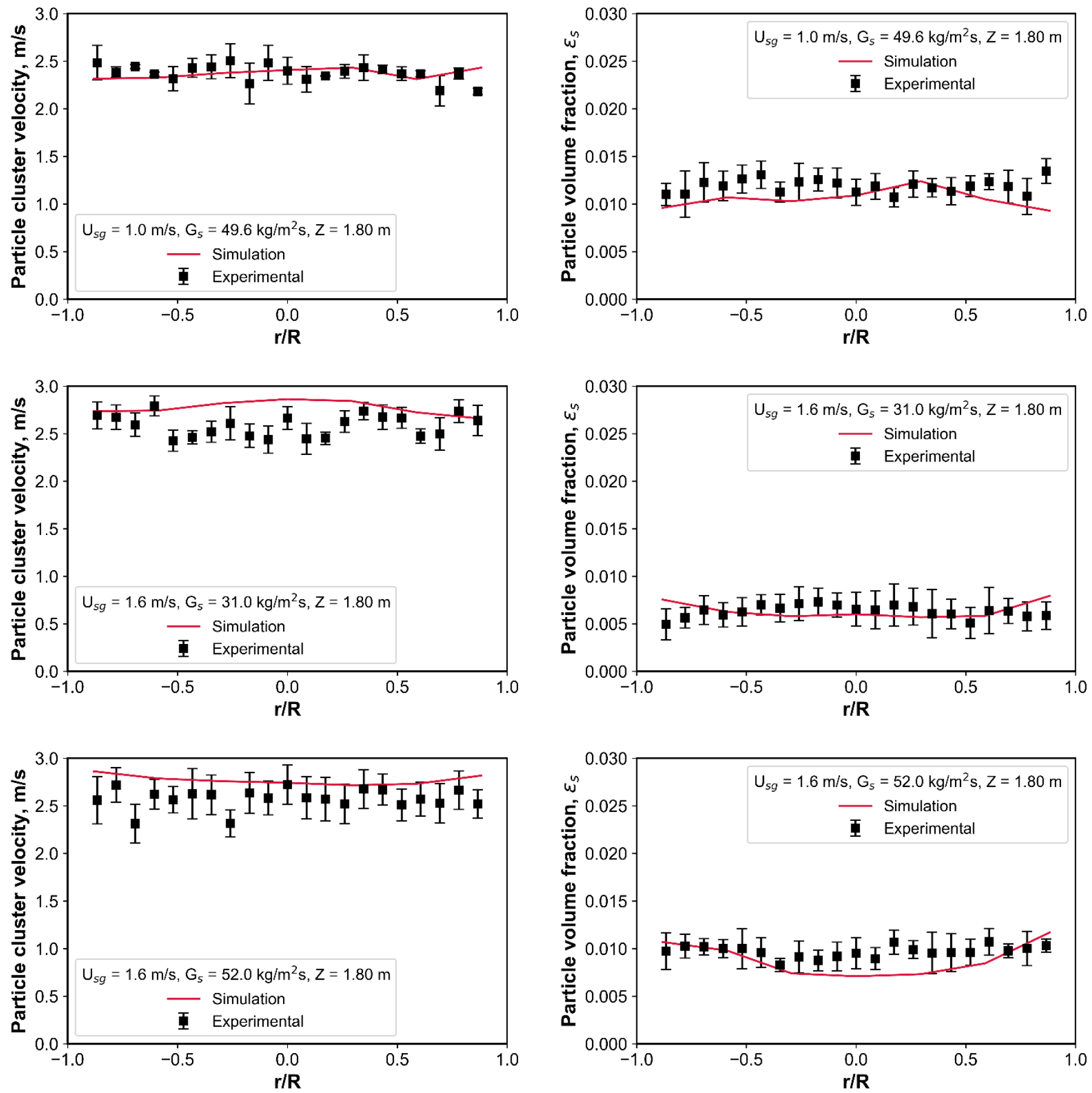

Figure 9. Radial Profiles of Cluster Velocities and Particle Volume Fractions at $Z=1.80 \mathrm{~m}$. Particle Cluster Geometry: Strand Shape.

One can thus again observe in Figure 9, that the Hybrid Barracuda Model is still quite adequate to represent the relatively uniform radial particle flow (cluster particle velocity and particle volumetric concentration), once the Hölzer-Sommerfeld drag correlation and the strand cluster geometry is selected. 
Furthermore, Table 4 provides a comprehensive description of the Hybrid Barracuda Model's ability to assess the relatively uniform radial profiles of both particle cluster velocity and particle volumetric concentration.

Table 4. Summary of the Radial Averaged Particle Cluster Velocity and Particle Volume Fraction for all Conditions at Three Axial Positions Studied.

\begin{tabular}{|c|c|c|c|c|c|c|}
\hline Condition & $U_{s g}(m / s)$ & $\mathrm{G}_{\mathrm{s}}\left(\mathrm{kg} / \mathrm{m}^{2} \mathrm{~s}\right)$ & $\bar{u}_{p, \exp }$ & $\bar{u}_{p, \text { sim }}$ & $\bar{\varepsilon}_{s, \exp }$ & $\bar{\varepsilon}_{s, s i m}$ \\
\hline \multicolumn{7}{|c|}{$\mathrm{Z}=0.2 \mathrm{~m}$ from air distributor } \\
\hline 1 & 1.0 & 31.4 & 2.019 & 1.956 & 0.0102 & 0.0103 \\
\hline 2 & 1.0 & 50.3 & 1.963 & 1.944 & 0.0153 & 0.0143 \\
\hline 3 & 1.6 & 31.2 & 2.421 & 2.280 & 0.0087 & 0.0089 \\
\hline 4 & 1.6 & 53.7 & 2.351 & 2.297 & 0.014 & 0.0135 \\
\hline \multicolumn{7}{|c|}{$\mathrm{Z}=0.4 \mathrm{~m}$ from air distributor } \\
\hline 5 & 1.0 & 30.2 & 2.104 & 2.162 & 0.0087 & 0.0087 \\
\hline 6 & 1.0 & 50.8 & 2.112 & 2.128 & 0.0129 & 0.0128 \\
\hline 7 & 1.6 & 30.5 & 2.519 & 2.522 & 0.0073 & 0.0077 \\
\hline 8 & 1.6 & 50.6 & 2.483 & 2.408 & 0.0117 & 0.0122 \\
\hline \multicolumn{7}{|c|}{$\mathrm{Z}=1.8 \mathrm{~m}$ from air distributor } \\
\hline 9 & 1.0 & 31.1 & 2.281 & 2.467 & 0.0071 & 0.0072 \\
\hline 10 & 1.0 & 49.6 & 2.376 & 2.372 & 0.0119 & 0.0105 \\
\hline 11 & 1.6 & 31.0 & 2.586 & 2.771 & 0.0063 & 0.0064 \\
\hline 12 & 1.6 & 52.0 & 2.576 & 2.774 & 0.0097 & 0.0089 \\
\hline
\end{tabular}

Table 4 shows the consistent closeness of the $\overline{\boldsymbol{u}}_{p, \text { exp }}$ and $\overline{\boldsymbol{u}}_{p, s i m}$, and the $\overline{\boldsymbol{\varepsilon}}_{\boldsymbol{s} \text { exp }}$ and $\overline{\boldsymbol{\varepsilon}}_{s, \text { sim }}$ with limited standard deviations, with this being true for all conditions considered. Thus, one can conclude that in a downer unit simulation a main challenge is to select the proper particle cluster geometry (either spherical or strand) and an appropriate particle drag correlation. This allows one to implement a valuable and applicable hybrid model, such as the one implemented with the Barracuda software in the present study. This can be done successfully in conjunction with availability of particle cluster size distribution data, obtained with the CREC-Optiprobes.

\section{Conclusions}

a) A Hybrid Barracuda Model was successfully implemented and validated for a downflow reactor unit, using CREC Optiprobes data obtained in a cold-flow unit.

b) The Hybrid Barracuda simulations were developed to establish both particle cluster drag correlations and particle cluster geometries (spherical, quasispheroidal, strand). It was shown that the Hölzer-Sommerfeld Model provides the best approximation of particle cluster velocities and particle volume fractions. 
c) The Hybrid Barracuda Model demonstrated as well, the need of considering spherical particle clusters in the acceleration region, evolving to particle strands while transitioning towards the stabilized section of the downer.

d) The Hybrid Barracuda simulations displayed relatively uniform radial profiles for both particle cluster velocities and particle volume fractions, with this being true at all axial positions considered.

\section{Supporting Information}

- Evaluation of the normal-to-wall restitution coefficient effect on particle cluster velocity.

\section{Acknowledgements}

The authors would like to acknowledge the funding from the scholarship granted to C. M. P. by CONACYT-Mexico and ICTI-Michoacan (No, 411626), as well as the Natural Sciences and Engineering Research Council of Canada (NSERC) Discovery Grant awarded to Hugo de Lasa. We would like to gratefully thank Florencia de Lasa who assisted with the editing of this paper.

\section{Abbreviations}

CFD, Computational Fluid Dynamics.

CPFD, Computational Particle Fluid Dynamics.

DEM, Discrete Element Method.

DNS, Direct Numerical Simulation.

FCC, Fluid Catalytic Cracking.

MP-PIC, Multiphase-Particle in Cell.

\section{Nomenclature}

$a$, major axis of spheroid cluster, $m$.

$b$, minor axis of spheroid cluster, $m$. $\dot{u}_{p}$, particle acceleration, $\mathrm{m} / \mathrm{s}^{2}$.

$u_{f}$, fluid velocity, $\mathrm{m} / \mathrm{s}$.

$u_{p}$, particle velocity, $\mathrm{m} / \mathrm{s}$.

$\bar{u}_{p}$, average particle velocity, $\mathrm{m} / \mathrm{s}$. 
$A_{c, s p h e r e}$ spherical cluster cross-sectional area, $m^{2}$.

$A_{c, \text { spheroid }}$, prolate spheroid cluster crosssectional area, $m^{2}$.

$A_{c, \text { strand }}$, cluster cross-sectional area normal to the flow for strand shape, $m^{2}$.

$C_{D}$, drag coefficient.

$d_{p}$, particle average diameter, $m$.

$D_{s}$, drag function, $\mathrm{s}^{-1}$.

$f$, particle distribution function.

$f_{D}$, collision probability distribution function.

$g$, gravity acceleration, $\mathrm{m} / \mathrm{s}^{2}$.

$G_{s}$, solid mass flux, $\mathrm{kg} / \mathrm{m}^{2} \mathrm{~s}$.

$K_{1}$, Stoke's shape factor.

$K_{2}$, Newton's shape factor.

$L$, cluster axial length, $\mathrm{m}$.

$m_{p}$, particle mass, $\mathrm{kg}$.

$n_{p}$, particle feed per average volume.

$N$, number of particles with average diameter in cluster.

$N_{\text {cells }}$, number of Eulerian cells.

$N_{p}$, total number of computational particles

$p$, pressure, $\mathrm{Pa}$.

$r / R$, dimensionless radial position.

$r_{p}$, particle radius, $\mathrm{m}$.

$r_{e q}$, radius of the equivalent sphere with same volume as cluster, $m$.

$R e_{p}$, Reynolds number defined for a spherical particle.

$t$, time, s. $\bar{u}_{p, \text { exp }}$, radial average experimental particle velocity, $\mathrm{m} / \mathrm{s}$.

$\bar{u}_{p, \text { sim }}$, radial average simulation particle velocity, $\mathrm{m} / \mathrm{s}$.

$U_{s g}$, superficial gas velocity, $\mathrm{m} / \mathrm{s}$.

$V_{\text {cell }}$, cell volume, $\mathrm{m}^{3}$.

$V_{c, s p h e r e}$, spherical cluster volume, $m^{3}$.

$V_{c, \text { spheroid }}$, cluster volume in prolate spheroid shape, $m^{3}$.

$V_{c, \text { strand }}$, cluster volume in strand shape, $\mathrm{m}^{3}$.

$x$, length dimension, $m$.

$Z$, axial position measured from the distributor, $m$.

\section{Greek symbols}

$\varepsilon_{f}$, fluid volume fraction.

$\varepsilon_{S}$, particle volume fraction.

$\varepsilon_{s, m f}$, particle volume fraction at minimum fluidization.

$\bar{\varepsilon}_{s, \text { exp }}$, radial average experimental particle volume fraction.

$\bar{\varepsilon}_{s, s i m}$, radial average simulation particle volume fraction.

$\rho_{f}$, fluid density, $\mathrm{kg} / \mathrm{m}^{3}$.

$\rho_{s}$, particle density, $\mathrm{kg} / \mathrm{m}^{3}$.

$\tau$, particle contact stress, $\mathrm{Pa}$.

$\tau_{f}$, fluid stress tensor, $P a$.

$\tau_{D}$, collision damping time, $s$.

$\phi_{\perp}$, cross-wise sphericity.

$\phi_{\| \mid}$, lengthwise sphericity.

$\phi$, standard sphericity. 


\section{References}

1. Abul-Hamayel MA. Comparison of Downer and Riser Based Fluid Catalytic Cracking Process at High Severity Condition: A Pilot Plant Study. Pet Sci Technol. 2004;22(5-6):475-490. doi:10.1081/LFT-120034183

2. Pannala S, Syamlal M, O'Brien TJ. Computational Gas-Solids Flows and Reacting Systems: Theory, Methods and Practice. Hershey, PA: Engineering Science Reference; 2010.

3. Zhu JX, Yu Z, Jin Y, Grace JR, Issangya A. Cocurrent downflow circulating fluidized bed (downer) reactors-a state of the art review. Can J Chem Eng. 1995;73:662677.

4. Wylie JJ, Koch DL. Particle clustering due to hydrodynamic interactions. Phys Fluids. 2000;12(5):964-970. doi:10.1063/1.870351

5. Krol S, Pekediz A, de Lasa HI. Particle clustering in down flow reactors. Powder Technol. 2000;108(1):6-20. doi:10.1016/S0032-5910(99)00196-5

6. Lu X, Li S, Du L, Yao J, Lin W, Li H. Flow structures in the downer circulating fluidized bed. Chem Eng J. 2005;112(1-3):23-31. doi:10.1016/j.cej.2005.06.002

7. Chen C, Werther J, Heinrich S, Qi HY, Hartge EU. CPFD simulation of circulating fluidized bed risers. Powder Technol. 2013;235:238-247. doi:10.1016/j.powtec.2012.10.014

8. Cocco R, Fullmer WD, Liu P. CFD-DEM - Modeling the small to understand the large. Chem Eng Prog. 2017;113(9):38-45.

9. Gidaspow D. Hydrodynamics of Circulating Fluidized Beds: Kinetic Theory Approach. Fluid VII. 1992;1.

10. Ropelato K, Meier HF, Cremasco MA. CFD study of gas-solid behavior in downer reactors: An Eulerian-Eulerian approach. Powder Technol. 2005;154(2-3):179-184. doi:10.1016/j.powtec.2005.05.005

11. Chalermsinsuwan B, Chanchuey T, Buakhao W, Gidaspow D, Piumsomboon P. 
Computational fluid dynamics of circulating fluidized bed downer: Study of modeling parameters and system hydrodynamic characteristics. Chem Eng J. 2012;189190:314-335. doi:10.1016/j.cej.2012.02.020

12. Cheng $\mathrm{Y}$, Wang $\mathrm{CH}$. Numerical study on coal gasification in the downer reactor of a triple-bed combined circulating fluidized bed. Ind Eng Chem Res. 2014;53(16):6624-6635. doi:10.1021/ie500013y

13. Tang, Yali; Peters, E.; Kuipers J. Direct numerical simulations of dynamic gas-solid suspensions. AlChE J. 2016;62(6):1958-1969. doi:10.1002/aic

14. Zhu HP, Zhou ZY, Yang RY, Yu AB. Discrete particle simulation of particulate systems: Theoretical developments. Chem Eng Sci. 2007;62(13):3378-3396. doi:10.1016/j.ces.2006.12.089

15. Zhang MH, Chu KW, Wei F, Yu AB. A CFD-DEM study of the cluster behavior in riser and downer reactors. Powder Technol. 2008;184(2):151-165. doi:10.1016/j.powtec.2007.11.036

16. Snider DM. An Incompressible Three-Dimensional Multiphase Particle-in-Cell Model for Dense Particle Flows. J Comput Phys. 2001;170:523-549. doi:10.1006/jcph.2001.6747

17. Abbasi A, Islam MA, Ege PE, de Lasa HI. CPFD Flow Pattern Simulation in Downer Reactors. AIChE J. 2013;59(5):1635-1647. doi:10.1002/aic.13956

18. Lanza A, de Lasa H. CPFD modeling and experimental validation of gas-solid flow in a down flow reactor. Comput Chem Eng. 2016;90(May):79-93.

19. Lanza A, de Lasa H. Scaling-up down flow reactors. CPFD simulations and model validation. Comput Chem Eng. 2017;101:226-242. doi:10.1016/j.compchemeng.2017.02.034

20. Medina-Pedraza C, de Lasa H. Cluster Acceleration and Stabilization in a Downflow Circulating Fluidized Bed Unit. Ind Eng Chem Res. 2020. doi:10.1021/acs.iecr.0c01397 
21. O'Rourke PJ, Snider DM. An improved collision damping time for MP-PIC calculations of dense particle flows with applications to polydisperse sedimenting beds and colliding particle jets. Chem Eng Sci. 2010;65(22):6014-6028. doi:10.1016/j.ces.2010.08.032

22. Ashraful Islam M, Krol S, de Lasa HI. The CREC-GS-Optiprobes and its focal region. Gas-solid flow measurements in down flow reactors. Chem Eng Sci. 2011;66(8):1671-1684. doi:10.1016/j.ces.2011.01.004

23. Chhabra RP, Agarwal L, Sinha NK. Drag on non-spherical particles: An evaluation of available methods. Powder Technol. 1999;101(3):288-295. doi:10.1016/S00325910(98)00178-8

24. Bagheri G, Bonadonna C. On the drag of freely falling non-spherical particles. Powder Technol. 2016;301:526-544. doi:10.1016/j.powtec.2016.06.015

25. Hölzer A, Sommerfeld M. New simple correlation formula for the drag coefficient of non-spherical particles. Powder Technol. 2008;184(3):361-365. doi:10.1016/j.powtec.2007.08.021

26. Li F, Song F, Benyahia S, Wang W, Li J. MP-PIC simulation of CFB riser with EMMS-based drag model. Chem Eng Sci. 2012;82:104-113. doi:10.1016/j.ces.2012.07.020

27. Kadyrov T, Li F, Wang W. Impacts of solid stress model on MP-PIC simulation of a CFB riser with EMMS drag. Powder Technol. 2019;354:517-528. doi:10.1016/j.powtec.2019.06.018

28. Ariyaratne WKH, Ratnayake C, Melaaen MC. Application of the MP-PIC method for predicting pneumatic conveying characteristics of dilute phase flows. Powder Technol. 2017;310:318-328. doi:10.1016/j.powtec.2017.01.048

29. Courant $\mathrm{R}$, Friedrichs $\mathrm{K}$, Lewy $\mathrm{H}$. On the Partial Difference Equations of Mathematical Physics. IBM J Res Dev. 1967;11(2):215-234. doi:10.1147/rd.112.0215

30. Lanza A, Islam MA, de Lasa H. Particle clusters and drag coefficients in gas-solid 
downer units. Chem Eng J. 2012;200-202:439-451. doi:10.1016/j.cej.2012.06.027

31. Ganser GH. A rational approach to drag prediction of spherical and nonspherical particles. Powder Technol. 1993;77(2):143-152. doi:10.1016/0032-5910(93)80051B 


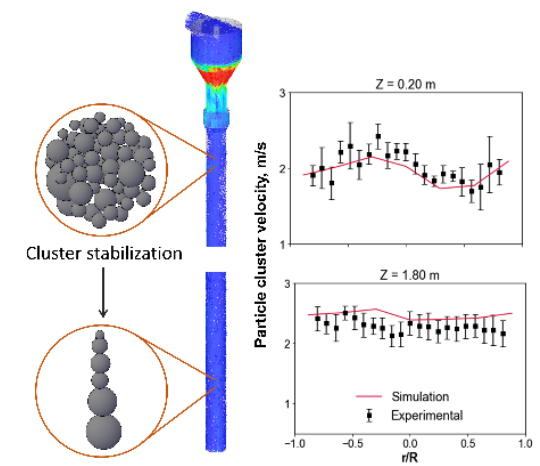

For Table of Contents Only 